\section{Brazil: world leader in anxiety and depression rates}

Rev. Bras. Psiquiatr. 2017;39:384

doi:10.1590/1516-4446-2017-2300

On February 23, the World Health Organization (WHO) published a survey ${ }^{1}$ on the two most common psychiatric illnesses affecting the world population: depressive and anxiety disorders. According to this survey, Brazil leads the world in prevalence of anxiety disorders and ranks fifth in depression rates.

Surveys of this kind are highly relevant, as they are often the basis of actions by the government and society and serve as guides for public policy-making for prevention and/or treatment. Whatever the use and attention that the data from this survey might have in the future, some reflections seem timely for an informed debate on this topic.

Compared to the preceding survey, released in $2005,{ }^{2}$ the latest WHO report describes an increase in the frequency of both depressive and anxiety disorders worldwide, pointing to population growth and increased longevity as contributing factors for the current picture. Although the latter factors are always involved in discussions about the prevalence of any disease, other potential confounders must be taken into account in this debate, including methodological discrepancies in diagnosis and data recording across countries, cultural differences in help-seeking behaviors, and differential availability and organization of mental health services, to name a few.

In 2007, the British Medical Journal invited two eminent researchers to answer the question "Is depression overdiagnosed?" in their section "Head to Head." Advocating that depression is overdiagnosed as a result of the medicalization of sadness, Parker ${ }^{3}$ stated that "a low threshold for diagnosing clinical depression [...] risks normal human emotional states being treated as illness, challenging the model's credibility and risking inappropriate management." In the opposite direction, Hickie ${ }^{4}$ wrote at the time that "From a health and economic perspective, we can give a clear answer - more adults are alive and well, and we can easily afford to treat more." Both authors supported their views with solid evidence.

Whatever the precise extent of the problem in Brazil and around the globe, it is already a collective issue, and the personal and financial burden of anxiety and depression demands prompt and coordinated action from the scientific community and government agencies.

In order to answer some of the questions raised by the WHO reports on mental health, research efforts should be directed to three main targets: 1) furthering our understanding of the etiological factors of anxiety and depressive disorders; 2) elucidating their social, cultural, and geographic determinants and impacts; and 3) increasing diagnostic and therapeutic accuracy. If the spread of these disorders is not checked - whether through preventative or therapeutic action - and the needs of affected individuals are not met, the productivity and social-security systems of many countries could be seriously jeopardized in the near future.

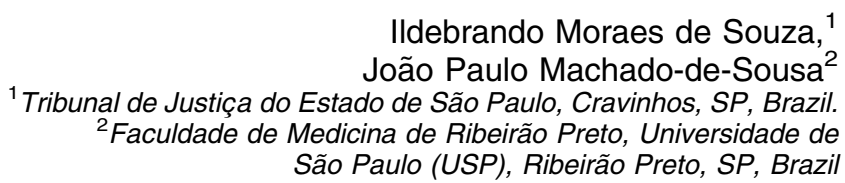

Submitted Apr 13 2017, accepted May 102017.

\section{Disclosure}

The authors report no conflict of interest.

\section{References}

1 World health Organization (WHO). Depression and other common mental disorders: Global Health Estimates [Internet]. 2017 Apr 7 [cited 2017 Jun 16]. www.who.int/mental_health/management/depression/ prevalence_global_health_estimates/en/.

2 World Health Organization (WHO). Mental Health Atlas 2005 [Internet]. 2005 [cited 2017 Jun 16]. www.who.int/mental_health/evidence/ mhatlas05/en/.

3 Parker G. Is depression overdiagnosed? Yes. BMJ. 2007;335:328.

4 Hickie I. Is depression overdiagnosed? No. BMJ. 2007;335:329.

\section{Schedule for Affective Disorders and Schizophrenia for School-Age Children - Present and Lifetime Version (K-SADS-PL), DSM-5 update: translation into Brazilian Portuguese}

Rev. Bras. Psiquiatr. 2017;39:384-386 doi:10.1590/1516-4446-2017-2317

The use of structured and semi-structured diagnostic instruments allows objective assessment of DSM categories by professionals involved in child and adolescent mental health evaluation, which has been essential to the advancement of clinical and epidemiological research in the field. Over the years, several semi-structured diagnostic instruments have been developed and tested for reliability and validity, such as the Diagnostic Interview for Children and Adolescents (DICA) ${ }^{1}$; the Development and Well-Being Assessment (DAWBA ${ }^{2}$; and the Schedule for Affective Disorders and Schizophrenia for Schoolage Children (K-SADS). ${ }^{3}$

The K-SADS is among the most widely used instruments in child and adolescent psychiatry. The first version was the K-SADS present state (K-SADS-P), developed by J. Puig-Antich and W. Chambers in 1978. Updates 